\title{
A 3D Printed, Constriction-Resistive Sensor for the Detection of Ultrasonic Waves
}

\author{
Saeb Mousavi ${ }^{1,2, a}$, Philippe Blanloeuil ${ }^{1, b}$, Thailammai Vinoth ${ }^{1, c}$, David Howard ${ }^{2, d}$, \\ Chun $\mathrm{H}$. Wang ${ }^{1, *}$
}

${ }^{1}$ School of Mechanical and Manufacturing Engineering, University of New South Wales, Sydney, NSW 2052, Australia
${ }^{2}$ The Robotics and Autonomous Systems Group, Commonwealth Scientific and Industrial Research Organization (CSIRO), Pullenvale, 4069, Australia
as.mousavianchehpoli@unsw.edu.au, bp.blanloeuil@unsw.edu.au, ct.vinoth@unsw.edu.au, ddavid.howard@csiro.au

${ }^{*}$ Corresponding author email: chun.h.wang@unsw.edu.au

\begin{abstract}
Keywords: 3D Printing, Constriction-Resistive Sensor, Ultrasound Sensor, Structural Health Monitoring
\end{abstract}

\begin{abstract}
Ultrasonic waves, either bulk waves or guided waves, are commonly used for non-destructive evaluation, for example in structural health monitoring. Traditional sensors for detecting ultrasonic waves include metallic strain gauges and piezoelectric ceramics. Recently piezoresistive nanocomposites have emerged as a promising sensor with high sensing range. In this paper, a constriction-resistive based sensor made from a graphene reinforced PLA filament is developed using a fused deposition modelling 3D printing approach as a novel type of ultrasonic sensor for structural health monitoring purposes. The sensor is made of very low-cost and recyclable thermoplastic material, which is lightweight and can be either directly printed onto the surface of various engineering structures, or embedded into the interior of a structure via fused filament fabrication 3D printing. These characteristics make this sensor a promising candidate compared to the traditional sensors in detecting ultrasonic waves for structural health monitoring. The printed sensors can detect ultrasonic signals with frequencies around $200 \mathrm{kHz}$, with good signal-to-noise ratio and sensitivity. When deployed between two adjacent printed tracks, and exploiting a novel kissing-bond mechanism, the sensor is capable of detecting ultrasonic waves. Several confirmatory experiments were carried out on this printed sensor to validate the capability of the printed sensor for structural health monitoring.
\end{abstract}

\section{Introduction}

Engineering structures can deteriorate to an unsafe or unrepairable level that can cause dramatic consequences such as endangering human life. Structural Health Monitoring (SHM) has come to prominence can detect defects in their early stage without risking the normal functionality of the structure under inspection. Ultrasonic SHM is an emerging and promising subfield, owing to its ability in monitoring multi-scale structures using both acoustic emission (AE) and guided ultrasonic waves (GUWs) approaches in a broad frequency regime [1-4].

In ultrasonic SHM, it is critical to develop a network of sensors to quantify and locate structural damage [5-7]. A broad range of sensors have been developed for this purpose, including lead zirconate titanate (PZT) wafers [8], carbon nanotube sensors [9,10], optical fibres [11], zinc oxide sensors [12], and graphene/polyvinylpyrrolidone (PVP) sensor [1]. Since a dense grid of sensors is usually required, not only the cost of production is a critical matter, but also sensors' weight and 
simplicity of fabrication becomes important. In this regard, a number of low-cost piezoresistive sensors composed of nanocomposite, flexible and low-density materials have been developed $[13,14]$.

In this paper, based on the authors' previous efforts on developing highly sensitive resistive strain sensors [15], a novel approach to strain sensing, called Constriction-Resistive (CR) sensing is applied for the detection of ultrasonic waves. The sensors are fabricated by a simple and low-cost fused deposition modelling (FDM) 3D printing technique and are extremely light weight. The morphology and electrical properties of the CR sensors are characterised. The CR sensor's response to ultrasonic wave is acquired and then validated by comparison with a commercial PZT transducer's response.

\section{Fabrication and Characterization of CR Sensors}

PLA-CNT serves as the sensing material. To fabricate the sensing material, PLA pellets and CNT were dissolved in dichloromethane $\left(\mathrm{CHCl}_{2}\right)$ solvent and then dried at room temperature to obtain the PLA-CNT composite with CNT mass fraction of $12 \%$. Then, the composite material was cut into pieces and extruded using a twin-screw extruder (Fig. 1a) to make filaments, which were then used to 3D print the sensor. The fabrication process for the sensors used in this work is fully explained in our previous paper $[15,16]$ and the step-by-step process is shown in Fig. 1 .
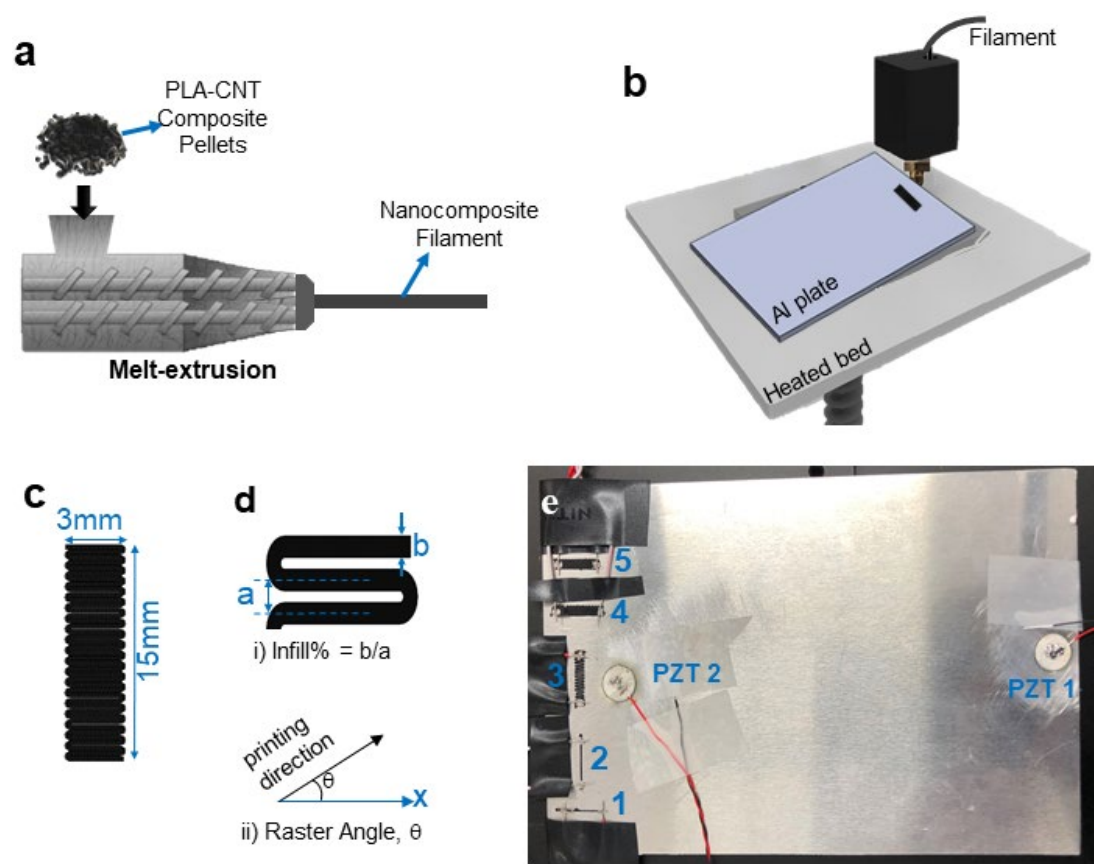

Fig. 1. Fabrication process of the CR sensors. (a) PLA pellets and CNT nanofillers are first solution-mixed and then fed into a twin-screw extruder. A filament is extruded through a nozzle at the end of the extruder. (b) The extruded filament is then used to 3D print the sensors on an aluminum plate. (c) dimensions of the printed sensors. (d) Illustrative definition of (i) infill density/ratio of $3 D$ printed sensor layer as ratio between track width, $b$, and the track spacing, $a$.

(ii) raster angle $(\theta)$ of $3 D$ printing. (e) An image of the 5 different printed sensors and two PZT transducers on the aluminum plate; 1) a single horizontal line, 2) a single vertical line, 3) a 90\% infill density sensor, 4 and 5) two 95\% infill density sensors. 
When two conducting materials come into contact, the true area of contact at the interface determines the true cross-section through which the electronic flow occurs. The flow of electrons or current bent together through these contact areas causes an increase in resistance beyond fully conducting surfaces. This contact resistance is also known as constriction resistance [15, 17]. The CR sensors were fabricated using an extremely simple FDM 3D printing technique. Three types of CR sensors and two single-track sensors were fabricated as shown in Fig. 1e. In 3D printing, infill ratio, which is defined as b/a, where $b$ is the width of the printed tracks and $a$ is the spacing between two neighbouring tracks, is an important parameter (Fig. 1d). By changing the infill density one can change the contact level of the printed neighbouring tracks, and therefore the level of constriction resistance existing between them. Changing the infill ratio affects the strength of the kissing bond existing between adjacent tracks. Utilising this parameter, CR sensors can be designed to display significantly higher sensitivity to deformation or vibrations compared to the solid sensors.

Optical images of the sensors with $85 \%, 90 \%, 95 \%$ and $100 \%$ infill densities are shown in Fig. 2. The SEM image of the fractured surface of the 3D printed PLA-CNT sensor (Fig. 2e) confirms the existence of CNT in the polymer matrix.
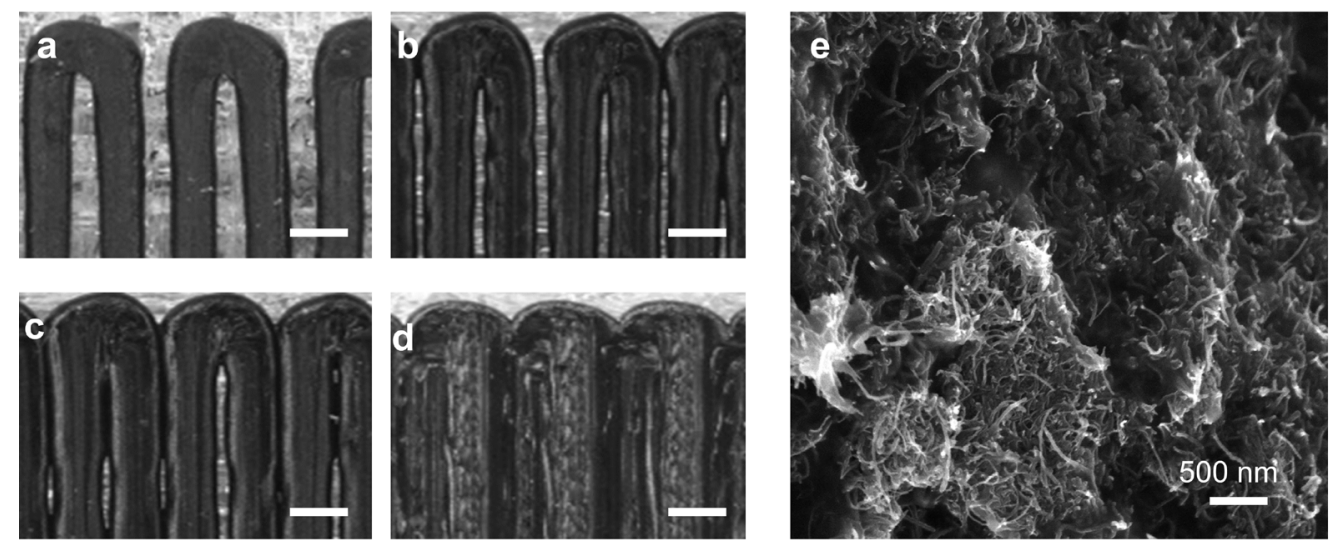

Fig. 2. Microstructure characterisation of the sensor materials. Microscopic images of the 3D printed PLA-CNT sensors with different infill densities (a) 85, (b) 90, (c) 95 and (d)100\%. The scale bars are $500 \mu \mathrm{m}$. (e) SEM image of fractured cross-sectional view of the $3 D$ printed sensor.

\section{Sensor Performance in Detection of Ultrasonic Wave}

To evaluate the performance of the sensors in the detection of ultrasonic waves, the sensors were $3 \mathrm{D}$ printed on an aluminium plate and were tested to see if they can detect the strain induced by a propagating wave in the plate generated by a lead zirconate titanate (PZT) piezoelectric transducer. Strain generated by the wave motion induces a resistance change in the sensors which is then transformed into voltage using a Wheatstone bridge and recorded through an oscilloscope. The schematic of the experimental setup is shown in Fig. 3.

The sensors' signals were recorded for a 3-cycles ultrasonic excitation wave with a frequency of $200 \mathrm{kHz}$. The results are shown in Fig. 4. Sensors 1 and 2 were unable to detect the source signal, whereas sensors 3, 4 and 5 were able to detect it. Since sensor 1 and 2 are just single tracks, there is no constriction resistance and therefore they do not show any sensitivity to acoustic excitation. On the other hand, sensor 3, 4 and 5 consist of weak contacts between printed tracks which makes them sensitive to the strain induced by the acoustic wave. 


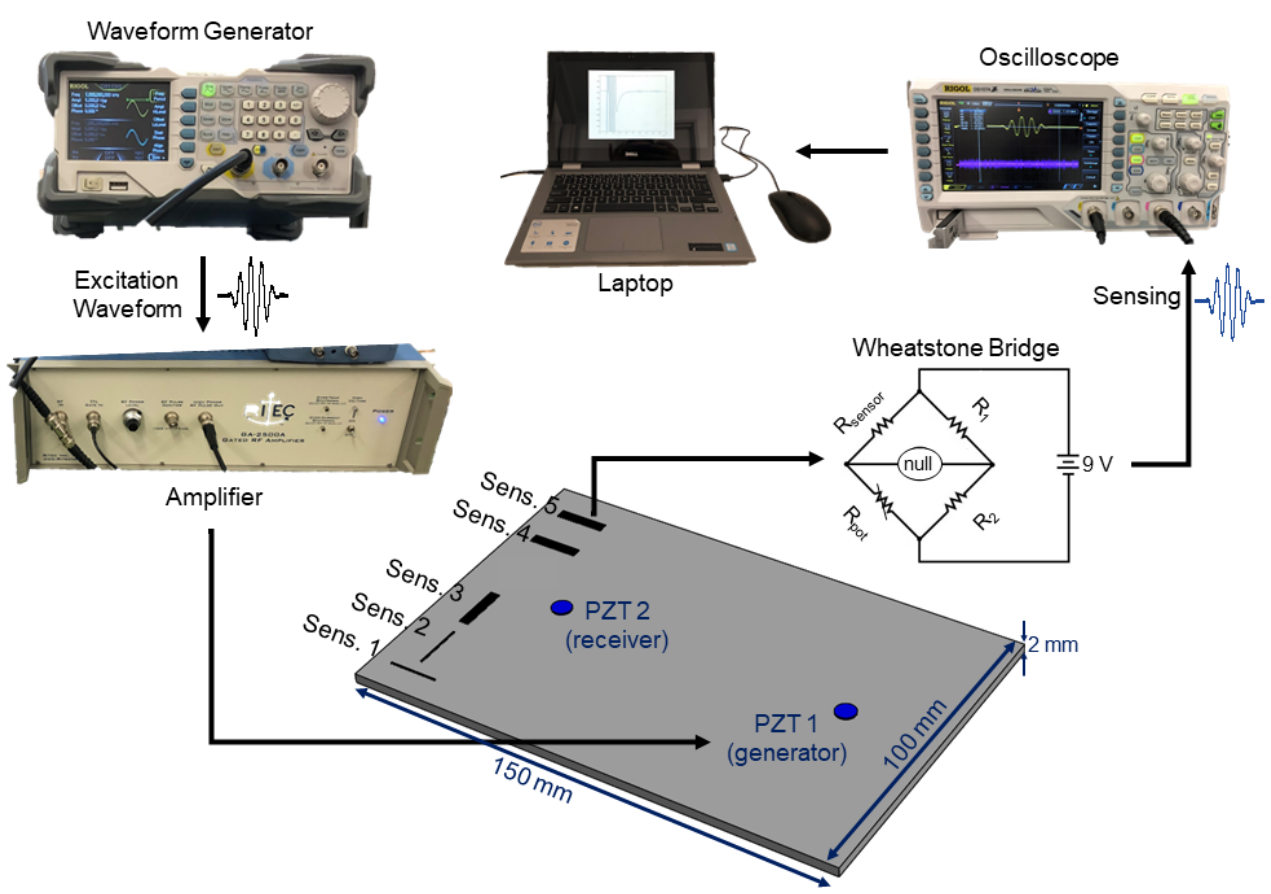

Fig. 3. Schematic of the experimental setup. The input signal is first generated in the desired form and then the signal is amplified using the amplifier and then transmitted to PZT 1 to generate the input ultrasonic wave. The sensors' output signal, which is resistance, is transformed into voltage using a Wheatstone bridge and then transmitted to oscilloscope for data acquisition and finally the data is saved on a laptop for further processing.
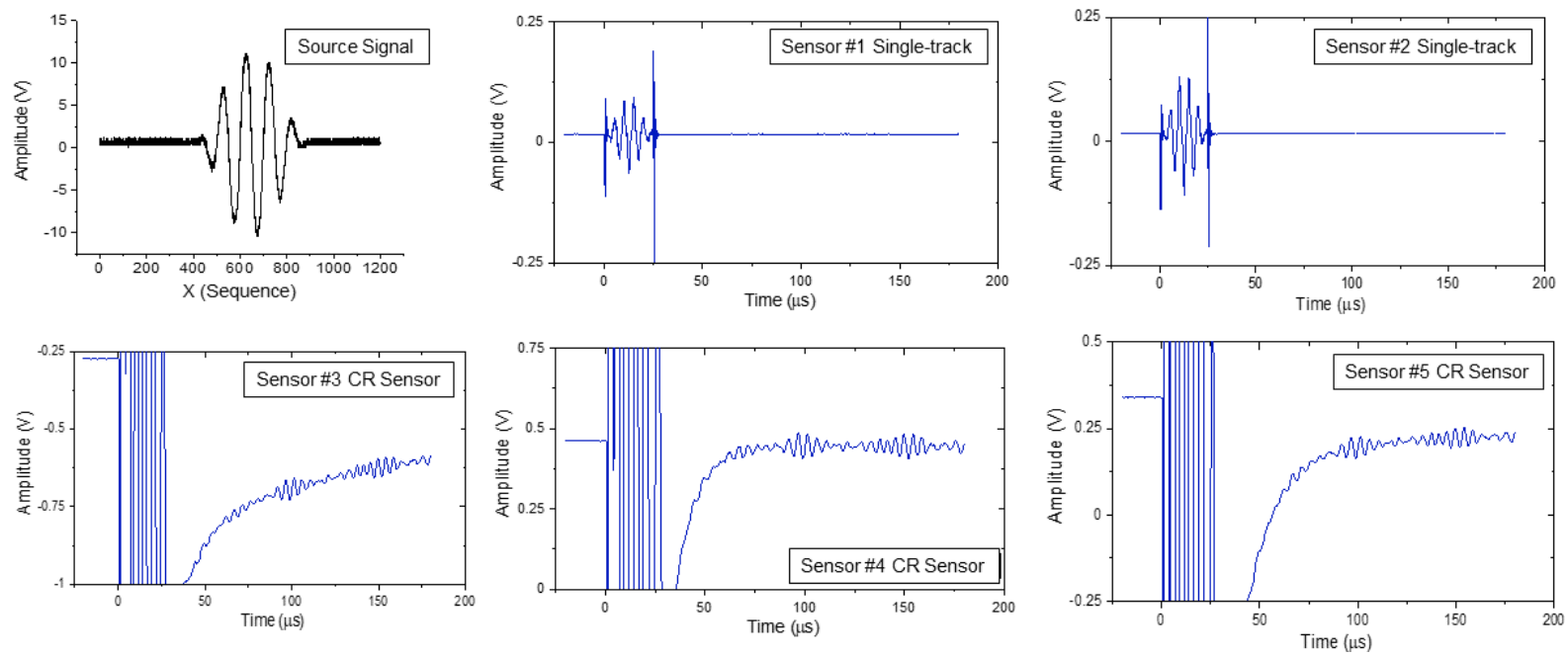

Fig. 4. CR Sensors' performance in detection of ultrasonic wave. Sensors 1 and 2 were unable to detect the source signal, whereas sensors 3, 4 and 5 were able to detect it.

In order to verify the sensors' response, a second PZT was attached to the plate near the location of the sensors as a receiver. The strain generated by the wave motion from PZT 1 induces a voltage change in PZT 2. The recorded signal for a 3-cycle $200 \mathrm{kHz}$ tone burst for PZT 2 and CR sensor \#4 are plotted in Fig. 5. As shown in this figure, the sensor's response is verified. Although the 
received signal by the sensor is weaker than the one obtained with PZT 2, the sensor can detect the input signal with a higher resolution and less noise compared to the PZT.

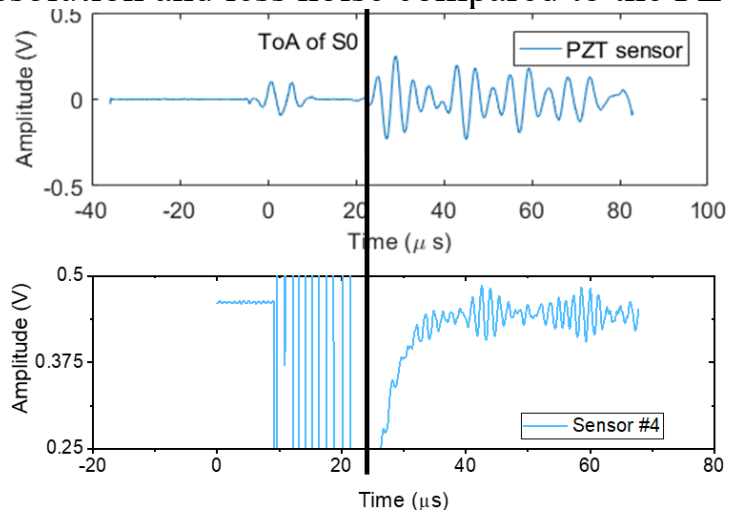

Fig. 5. Comparison of the CR sensor's response with PZT 2. This verifies the sensor's response although the received signal by the sensor is weaker than the one obtained by PZT 2.

\section{Conclusions}

We presented a novel design for damage detection and SHM of component. Our 3D printed constriction-resistive strain sensors are responsive to tiny deformations under acoustic vibration. Infill ratio has been found to play a key role in the sensitivity of the sensors. As shown in Fig. 5, the sensors were able to successfully predict the source signals with minimal error. The limitation of the current sensors is their low sensitivity and we are currently conducting more experiments to enhance the sensitivity of the CR sensors. Furthermore, a full study for better understanding of the sensing mechanism is undergoing.

Considering the rapid development of 3D printing techniques, this research paves the way for low cost and accessible techniques to enable in-situ and offline SHM of damaged components.

\section{Summary}

In this paper, a constriction-resistive ultrasonic wave sensor is presented that is fabricated through simple FDM 3D printing and can be employed for structural health monitoring applications.

\section{References}

[1] W. Cao, P. Zhou, Y. Liao, X. Yang, D. Pan, Y. Li, B. Pang, LM. Zhou, Z. Su, A Spray-on, Nanocomposite-Based Sensor Network for in-Situ Active Structural Health Monitoring. Sensors 19, 9 (2019) 2077. https://doi.org/10.3390/s19092077

[2] N. Hu, T. Shimomukai, H. Fukunaga, Z. Su, Damage identification of metallic structures using A0 mode of lamb waves. Struct. Health Monit. 7 (2008) 271-285.

https://doi.org/10.1177/1475921708090566

[3] H. Mei, M.F. Haider, R. Joseph, A. Migot, V. Giurgiutiu, Recent advances in piezoelectric wafer active sensors for structural health monitoring applications. Sensors 19 (2019) 383. https://doi.org/10.3390/s19020383

[4] M.F. Haider, V. Giurgiutiu, Analysis of axis symmetric circular crested elastic wave generated during crack propagation in a plate: A Helmholtz potential technique. Int. J. Solids Struct. 134 (2018) 130-150. https://doi.org/10.1016/j.ijsolstr.2017.10.035

[5] W. Ostachowicz, R. Soman, P. Malinowski, Optimization of sensor placement for structural health monitoring: A review. Struct. Health Monit. 18, 3 (2019), 963-988.

https://doi.org/10.1177/1475921719825601 
[6] T. Wandowski, P. Malinowski, W. Ostachowicz, Circular sensing networks for guided waves based structural health monitoring. Mech. Syst. Sig. Process. 66 (2016) 248-267. https://doi.org/10.1016/j.ymssp.2015.05.001

[7] M. Salmanpour, Z. Sharif Khodaei, M. Aliabadi, Transducer placement optimisation scheme for a delay and sum damage detection algorithm. Struct. Control Health Monit. 24 (2017) e1898. https://doi.org/10.1002/stc. 1898

[8] R. Takpara, M. Duquennoy, M. Ouaftouh, C. Courtois, F. Jenot, M. Rguiti, Optimization of PZT ceramic IDT sensors for health monitoring of structures. Ultrasonics 79 (2017) 96-104. https://doi.org/10.1016/j.ultras.2017.04.007

[9] A. D’Alessandro, M. Rallini, F. Ubertini, A.L. Materazzi, J.M. Kenny, Investigations on scalable fabrication procedures for self-sensing carbon nanotube cement-matrix composites for SHM applications. Cem. Concr. Compos. 65 (2016) 200-213. https://doi.org/10.1016/j.cemconcomp.2015.11.001

[10] I. Kang, M.J. Schulz, J.H. Kim, V. Shanov, D. Shi, A carbon nanotube strain sensor for structural health monitoring. Smart Mater. Struct. 15 (2006) 737-748.

https://doi.org/10.1088/0964-1726/15/3/009

[11] T.H. Loutas, P. Charlaftis, A. Airoldi, P. Bettini, C. Koimtzoglou, V. Kostopoulos, Reliability of strain monitoring of composite structures via the use of optical fiber ribbon tapes for structural health monitoring purposes. Compos. Struct. 134 (2015) 762-771.

https://doi.org/10.1016/j.compstruct.2015.08.100

[12] H. Gullapalli, V.S.M. Vemuru, A. Kumar, A. Botello-Mendez, R. Vajtai, M. Terrones, S. Nagarajaiah, P.M. Ajayan, Flexible Piezoelectric ZnO-Paper Nanocomposite Strain Sensor. Small 6 (2010) 1641-1646. https://doi.org/10.1002/smll.201000254

[13] Z. Zeng, M. Liu, H. Xu, Y. Liao, F. Duan, L. Zhou, H. Jin, Z. Zhang, Z. Su, Ultra-broadband frequency responsive sensor based on lightweight and flexible carbon nanostructured polymeric nanocomposites. Carbon 121 (2017) 490-501. https://doi.org/10.1016/j.carbon.2017.06.011 [14] Z. Zeng, M. Liu, H. Xu, Y. Liao, F. Duan, L. Zhou, H. Jin, Z. Zhang, Z. Su, A coatable, light-weight, fast-response nanocomposite sensor for the in situ acquisition of dynamic elastic disturbance: From structural vibration to ultrasonic waves. Smart Mater. Struct. 25 (2016) 065005. https://doi.org/10.1088/0964-1726/25/6/065005

[15] S. Mousavi, D. Howard, F. Zhang, J. Leng, CH. Wang, Direct 3D Printing of Highly Anisotropic, Flexible, Constriction-Resistive Sensors for Multidirectional Proprioception in Soft Robots. ACS Applied Materials \& Interfaces 12, 13 (2020), 15631-15643. https://doi.org/10.1021/acsami.9b21816

[16] S. Mousavi, D. Howard, S. Wu, C.H. Wang, An ultrasensitive 3D printed tactile sensor for soft robotics, arXiv preprint, arXiv:1810.09236.

[17] Murty, Y. V. Electrical and Electronic Connectors: Materials and Technology. In Encyclopedia of Materials: Science and Technology; Buschow, K. H. J., Cahn, R. W., Flemings, M. C., Ilschner, B., Kramer, E. J., Mahajan, S., Veyssière, P., Ed.; Elsevier, 2001; pp 2483-2494. https://doi.org/10.1016/B0-08-043152-6/00450-2 